\title{
Time to eat? The relationship between food security and food-related time use
}

\author{
Timothy KM Beatty ${ }^{1}$, M Susie Nanney ${ }^{2}$ and Charlotte Tuttle ${ }^{1, *}$ \\ 'Department of Applied Economics, University of Minnesota, 313 Ruttan Hall, 1994 Buford Avenue, St. Paul, \\ MN 55108, USA: ${ }^{2}$ Department of Family Medicine \& Community Health, University of Minnesota, Minneapolis, \\ MN, USA
}

Submitted 9 April 2012: Final revision received 14 November 2012: Accepted 6 December 2012: First published online 23 January 2013

\begin{abstract}
Objectives: In the present analysis, we seek to establish a relationship between time spent on food-related activities and food security status as well as between time spent on these activities and Supplemental Nutrition Assistance Program (SNAP, formerly called the Food Stamp Program) participation and benefit level. Design: After matching similar households using Coarsened Exact Matching, we estimate the relationship between food-related time, food insecurity and SNAP participation and benefit level using a comprehensive data set that combines two subsets of the Current Population Survey from years 2004-2010: the Food Security Supplement and the American Time Use Survey.

Setting: City, suburban and rural areas of the USA.

Subjects: Non-institutionalized US population over the age of 15 years. Total sample size is 10247 households.

Results: In single households, food insecurity and SNAP participation are associated with $20 \%$ more time in meal preparation and 13\% less time eating. Similarly, in married households, SNAP participation and benefit level are associated with $32 \%$ less time in meal preparation while food insecurity is associated with $17 \%$ less time eating and 14\% less time in grocery shopping.

Conclusions: A significant relationship exists between time spent on food-related activities and food insecurity and SNAP. This implies that federal and state government may need to consider the time constraints many low-income households face when reforming food assistance programmes.
\end{abstract}

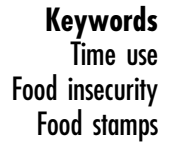

Keywords

Food insecurity

Food stamps
Because many low-income households are headed by single, working adults with children, these households often face time as well as budget limitations ${ }^{(1)}$. Similar to households facing budget limitations, households facing time limitations may also be vulnerable to nutritional consequences due to a possible lack of access to sufficiently healthy and well-balanced meals. While federal nutrition programmes such as the Supplemental Nutrition Assistance Program (SNAP, formerly called the Food Stamp Program) assist households facing limited budgets, it is not apparent that these same programmes address the time constraints some households face. However, SNAP does provide nutrition assistance benefits to eligible low-income households that can be used to purchase foods from authorized food retailers, thereby providing a safety net. In addition, state agencies can be reimbursed for providing optional nutrition education. But nutrition education programmes do not necessarily address time constraints some low-income households face. As a result, participants may not experience the maximum programme benefits when time constraints are not fully considered.
Few studies have explored how food-insecure households or SNAP participants allocate time towards household meal production and other food-related activities. Self-efficacy, central to social cognitive theory, has been explored as a contributor to diet quality and food insecurity and may be related to time management. High levels of self-efficacy, or belief in one's ability to control and improve one's life for the better, have been found to be correlated with improved diet quality and nutrition $^{(2-4)}$ while low levels of self-efficacy have been correlated with food insecurity ${ }^{(5)}$. In other words, those with high levels of self-efficacy may be more effective when allocating time and money towards meal plans. One such result of increased self-efficacy may be efficient or effective time use in food preparation.

Previous studies have indicated that following the Thrifty Food Plan (TFP), which outlines low-cost, healthy diets for SNAP participants, may be related to increased time spent in food preparation. Rose ${ }^{(6)}$ found that following the TFP requires $2 \cdot 3 \mathrm{~h}$ of time in food preparation daily. This implies that many participating households that face 
time constraints may be less able or more challenged to achieve nutritious diets. Davis and $\mathrm{You}^{(7)}$ calculated the proportion of total meal cost that is attributable to preparation time. They found that preparation time cost is $63 \%$ of total meal cost for households that follow the TFP as compared with $35 \%$ of total cost for the general population. In other words, households following the TFP appear to spend significantly more time in food preparation than the general population. Finally, Hamermesh ${ }^{(8)}$ found that time spent related to food production was positively associated with food expenditure. Therefore, substituting goods, or ingredients, in order to make up for lack of preparation time may be challenging for those also facing budgetary obstacles. As a result, if a household lacks time for meal preparation, increasing food expenditure may not prevent households from facing food insecurity.

Little research has directly examined household time use in food-related activities. The aim of the present paper is to estimate the relationship between food insecurity and time spent in food-related activities, as well as between SNAP participation and time spent in food-related activities, using data from the Current Population Survey-Food Security Supplement (FSS) and the American Time Use Survey (ATUS).

\section{Data and methods}

In order to investigate whether time spent in food-related activities is related to SNAP participation and food insecurity, we combine two data sources to develop a comprehensive data set. This data set combines the $\operatorname{ATUS}^{(9)}$ and the FSS ${ }^{(10)}$. Combining these data sets, we are able to observe whether a relationship exists between time spent on food-related activities and SNAP participation and benefit level as well as between time spent on food-related activities and food insecurity.

Because the ATUS and the FSS are subsets of the Current Population Survey, a monthly survey conducted by the Bureau of Labor Statistics, we are able to merge the data sets at the person level. As a result, we can capture information from the same person appearing in both data sets. The ATUS contains specific information on daily activities of the respondent, who reports these activities for a 'sample day' as well as the amount of time he/she spends in each activity. Other information collected in the FSS and ATUS include employment, income and demographic characteristics such as age, race and gender. In the present study, we focus on activities related to food acquisition and preparation, and analyse data from the years 2004 to 2010 .

\section{Construction of variables}

Independent variables

We construct our independent variables using information from the FSS. We simplify each of the following variables to allow a clear interpretation of each variable. We log transform all continuous variables given that each variable is bounded by zero and positively skewed. By log transforming these variables, the distribution of the data is more symmetric.

SNAP participation. We construct a variable 'SNAP Participation', where the value of one indicates that the respondent has participated in SNAP over the past 12 months and zero indicates that the respondent did not participate in the programme over the past 12 months.

SNAP benefits. To explore the relationship between SNAP benefit amount and time spent on food-related activities, we construct the variable 'SNAP Benefits' as the natural logarithm of the monthly SNAP benefit amount in dollars currently received by the respondent reported in the FSS. If the amount reported is zero, we recode 'SNAP Benefits' to equal zero.

By estimating 'SNAP Participation' and 'SNAP Benefits' separately, we are able to distinguish between the extensive and intensive margin. Including 'SNAP Participation' reveals whether participating in the SNAP matters; including 'SNAP Benefits' demonstrates the relationship between the dependent variable and different levels of benefits.

Food insecurity. The FSS includes an eighteen-item questionnaire that classifies respondent households into different categories of food security: food secure or marginally food secure, low food secure and very low food secure. Using this measure, we construct a dummy variable 'Food Insecure', where the value of one indicates that a household is food insecure, or low or very low food secure, and zero indicates food secure or marginally food secure.

Other explanatory variables. Our demographic variables include: the number of children under the age of 18 years in the household; the number of adults in the household; female; age; race; income represented by sixteen categories of income level, ranging from 0 to \$US 5000 up to \$US 150000 and above; employment; and Metropolitan Statistical Area status, which categorizes households as residing in a metropolitan area (defined as a city with a population greater than one million) or in a non-metropolitan area (with a population under one million). We also control for unobservable state and year factors by including state and year fixed effects.

\section{Dependent variables}

For our dependent variables, we construct variables capturing how households use time from the ATUS. Each outcome variable is constructed as the natural logarithm of each specific time-use variable. We include any variables related to food-related activities.

Meal preparation time. The variable 'Meal Prep' represents the natural logarithm of the number of minutes 
the respondent spends in meal or snack planning, cooking, presentation and clean up.

Grocery store time. The variable 'Grocery' represents the natural logarithm of the number of minutes the respondent spends food shopping at a grocery store.

Non-grocery store time. The variable 'Non-Grocery' represents the natural logarithm of the number of minutes the respondent spends buying food from locations other than a grocery store. These include restaurants, fast-food establishments, convenience stores and vending machines.

Eating time. The variable 'Eating' represents the natural logarithm of the number of minutes the respondent spends eating.

\section{Empirical model}

Using our combined data set we estimate a linear regression model:

$$
T U_{i s t}=F I_{i s t} \beta_{1}+S N A P_{i s t} \beta_{2}+X_{i} \beta_{3}+u_{s}+v_{t}+\varepsilon_{i s t},
$$

where $T U_{\text {ist }}$ represents the time households spend in the four food-related activities described above, where the household is represented by $i$ in state $s$ in year $t ; F I_{\text {ist }}$ represents household food insecurity; $S N A P_{i s t}$ represents SNAP participation or benefit level; $X_{i}$ represents household demographics included in the model; $u_{s}$ represents state fixed effects; and $v_{t}$ represents year fixed effects. $\varepsilon_{i s t}$ represents the error term that captures unobservable determinants of time spent in each food-related activity. Because previous research has indicated that married and single households allocate time differently, we estimate these households separately ${ }^{(11)}$. These models are estimated using ordinary least squares.

\section{Data preparation}

The SNAP literature indicates that including SNAP participation as an explanatory variable introduces endogeneity due to selection bias ${ }^{(12,13)}$. Endogeneity occurs when unobservable characteristics affect whether a respondent chooses to participate in the SNAP as well as how the respondent spends time in food-related activities, food security status and other explanatory variables. Likewise, unobservable characteristics, such as self-efficacy, that determine food security status of households may also be correlated with other explanatory variables as well as SNAP participation.

To mitigate, but not solve, the endogeneity problem, we use the Coarsened Exact Matching method ${ }^{(14)}$ in order to improve the balance of data. This forces the distribution of observed explanatory variables between the treated and control groups to be similar. For our analysis, we coarsen a number of explanatory variables including income, employment, race, gender, number of children and residence status. We then match on the treatment group, 'SNAP Participation'. Following the method of Ho et $a l^{(15)}$, we discard any unmatched data or weighting measures after matching the data and run our original parametric method on the pruned data. Unmatched households dropped from analysis tended to have higher incomes than the matched households. Of the unmatched data we dropped, 58\% had incomes above \$US 75000 and nearly 70\% had incomes above \$US 60000 . On the other hand, $14 \%$ of the matched households had income over \$US 75000. Moreover, the majority of the households excluded were food secure and did not participate in SNAP. Overall, we discarded 3191 observations from the single household sub-sample and 6131 from the married household sub-sample.

\section{Results}

Table 1 contains descriptive statistics for our combined data set. Our sample contains households of all ages and income levels. However, due to the Coarsened Exact Matching process, a majority of the respondents have

Table 1 Summary statistics: merged ATUS and FSS data sets, USA, 2004-2010

\begin{tabular}{|c|c|c|c|c|c|c|}
\hline \multirow[b]{2}{*}{ Variable } & \multicolumn{3}{|c|}{ Single } & \multicolumn{3}{|c|}{ Married } \\
\hline & Mean & SD & $n$ & Mean & SD & $n$ \\
\hline SNAP participation & $0 \cdot 135$ & $0 \cdot 342$ & 842 & 0.063 & $0 \cdot 242$ & 271 \\
\hline Food insecurity status & $0 \cdot 167$ & $0 \cdot 372$ & 1098 & $0 \cdot 120$ & $0 \cdot 324$ & 517 \\
\hline Average monthly benefit level of participant households (\$US) & $240 \cdot 25$ & $147 \cdot 09$ & & $174 \cdot 88$ & $137 \cdot 18$ & \\
\hline White & 0.773 & 0.419 & 5105 & 0.910 & $0 \cdot 274$ & 3976 \\
\hline Black & $0 \cdot 218$ & $0 \cdot 413$ & 1435 & 0.075 & $0 \cdot 264$ & 326 \\
\hline American Indian & 0.002 & 0.046 & 15 & 0.001 & 0.037 & 6 \\
\hline Asian & 0.000 & 0.000 & 0 & 0.002 & 0.040 & 7 \\
\hline Employed & 0.51 & $0 \cdot 49$ & 3389 & $0 \cdot 60$ & $0 \cdot 48$ & 2598 \\
\hline Female & 0.65 & 0.48 & 4246 & 0.55 & 0.50 & 2391 \\
\hline Age (years) & $48 \cdot 58$ & $20 \cdot 66$ & & $46 \cdot 77$ & $15 \cdot 24$ & \\
\hline No. of children & 0.54 & 0.94 & & $1 \cdot 14$ & $1 \cdot 16$ & \\
\hline No. of adults & $1 \cdot 45$ & 0.81 & & $2 \cdot 16$ & 0.54 & \\
\hline Hourly wage (\$US) & $12 \cdot 42$ & $6 \cdot 32$ & & $14 \cdot 36$ & $7 \cdot 10$ & \\
\hline Hours worked per week & $38 \cdot 58$ & $13 \cdot 66$ & & $40 \cdot 08$ & $13 \cdot 37$ & \\
\hline Observations & \multicolumn{2}{|c|}{6590} & \multicolumn{4}{|c|}{4331} \\
\hline
\end{tabular}

ATUS, American Time Use Survey; FSS, Current Population Survey-Food Security Supplement; SNAP, Supplemental Nutrition Assistance Program. 
incomes below \$US 50000 , a household size of three or more and at least one child present in the household. By using Coarsened Exact Matching as opposed to restricting the data to households with incomes at $130 \%$ of the Federal Poverty Guideline, we are able to address selection issues that tend to affect SNAP and food insecurity studies that restricting the sample would not address. SNAP participation is about $13 \%$ for single households and about $6 \%$ for married households. About $17 \%$ of single households and $12 \%$ of married households indicate behaviour consistent with food insecurity. Although these figures are below the national average for SNAP participation and food insecurity, it is important to note that merging the FSS with the ATUS results in a drop in SNAP participants as well as food-insecure households. This may be because these households are less likely to respond to the ATUS. Of households participating in SNAP, the average benefit level was about \$US 240/month for married households and \$US 175/month for single households. The maximum benefit level was $\$$ US $450 /$ month.

Our main findings are summarized in Tables 2 and 3. Each table represents two separate independent variables: Table 2 contains the results of the regression models which include only SNAP Benefits and Food Insecure; Table 3 includes the results of the regression models which include only SNAP Participation and Food Insecure. Results of SNAP Benefits and SNAP Participation are adjusted for Food Insecure. Likewise, results for Food Insecure are adjusted for SNAP Benefits and SNAP Participation.

Table 2 contains the results for SNAP benefit level. We find that a $1 \%$ increase in SNAP Benefits is associated with $7 \%$ fewer minutes in meal preparation time. We calculate that the average respondent spends $35 \mathrm{~min}$ in food preparation daily. Suppose SNAP benefits increase by $10 \%$, this translates to $24 \mathrm{~min}$ less in preparation time. Similarly, a $1 \%$ increase in household SNAP benefit level in married households is associated with $2 \%$ fewer minutes in non-grocery food shopping. Because it is possible that households do not shop for food daily, the average time spent on food shopping, both grocery and non-grocery, may not accurately reflect the amount of time households spend per week or month. In other words, because the survey is a $24 \mathrm{~h}$ recall period, it is possible that the respondent did not shop for food at the grocery store or any other location on the day of the survey. This is explicit in the data, where $84 \%$ of the households report $0 \mathrm{~min}$ spent in grocery shopping and $88 \%$ report $0 \mathrm{~min}$ in non-grocery food shopping. Therefore, including all households in the calculation of average daily food shopping would underestimate actual food shopping time of the household. We calculate the average time spent in these activities using only households who report a positive amount of time food shopping. Average non-grocery shopping time for households is $10.5 \mathrm{~min}$. Accordingly, a

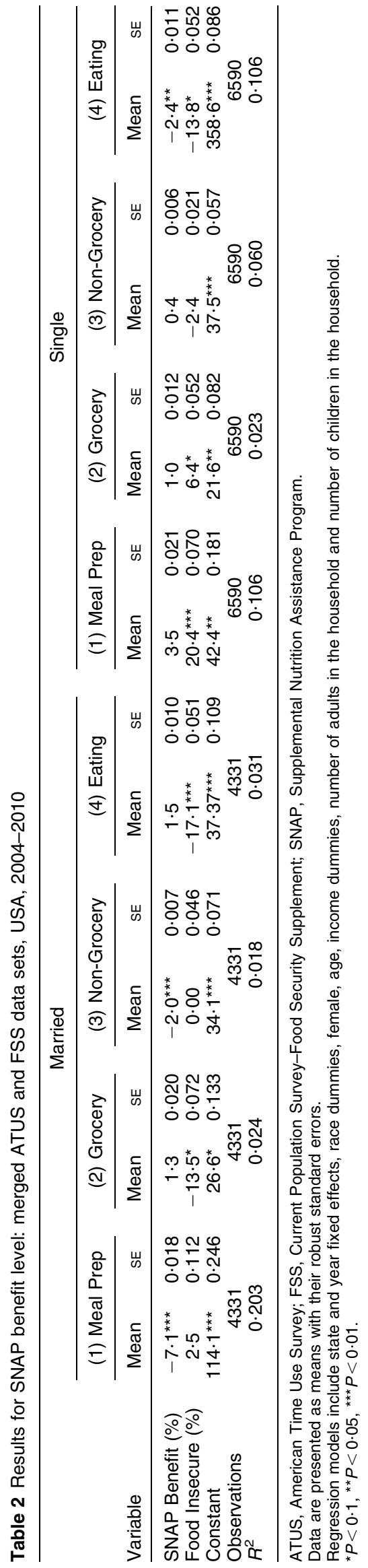




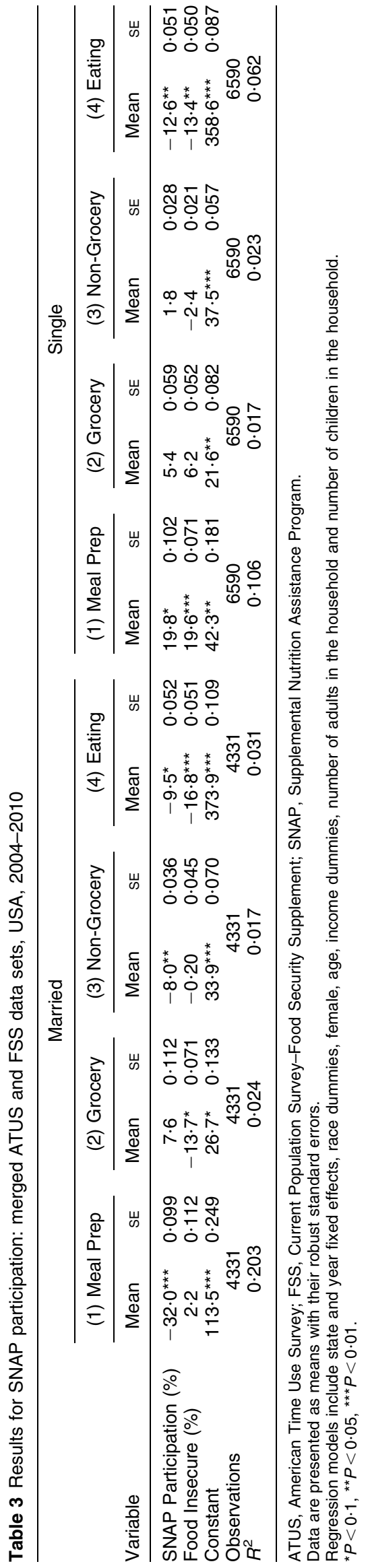

$10 \%$ increase in SNAP benefits translates to 2 min less in non-grocery food shopping.

In married households, Food Insecure is associated with less time grocery shopping and eating. Average grocery shopping time for households is $45 \mathrm{~min}$. Therefore, being food insecure is related to nearly $14 \%$ less time shopping at a grocery store, which translates to about $6.5 \mathrm{~min}$ less. Being food insecure is also related to $17 \%$ less time in eating. If the average household spends about $70 \mathrm{~min}$ eating daily, this translates to $12 \mathrm{~min}$ less in eating.

In single households, a $1 \%$ increase in SNAP Benefits is associated with $2 \cdot 4 \%$ less time in eating. Therefore, a $10 \%$ increase in benefits is associated with 17 min less in eating. Being food insecure is associated with $20 \%$ more time in meal preparation, $6.4 \%$ more time in grocery shopping and $13.6 \%$ less time in eating; these results translate to $7 \mathrm{~min}$ more in meal preparation, $3 \mathrm{~min}$ more in grocery shopping and nearly $10 \mathrm{~min}$ less in eating.

Table 3 contains results for SNAP Participation. In married households, SNAP Participation is associated with $32.0 \%$ less time in meal preparation, $8.0 \%$ less time in non-grocery food shopping and $9.5 \%$ less time in eating. These results translate to nearly $15 \mathrm{~min}$ less in meal preparation, about $1 \mathrm{~min}$ less in non-grocery food shopping and $8 \mathrm{~min}$ less in eating. Similarly, Food Insecure is correlated with nearly $14 \%$ fewer minutes in grocery shopping and almost $17 \%$ fewer minutes in eating in married households. This translates to 6 min less in grocery shopping and $12 \mathrm{~min}$ less in eating.

In single households, SNAP Participation is associated with nearly $20 \%$ more time in meal preparation and $12.6 \%$ less time in eating. This suggests that single households, on average, spend $7 \mathrm{~min}$ more on food preparation and nearly $9 \mathrm{~min}$ less on eating daily. The Food Insecure variable yields nearly identical results. In single households, being food insecure is associated with about $20 \%$ more time in meal preparation and $13.4 \%$ fewer minutes in eating. In other words, on a daily basis, food-insecure households tend to spend $7 \mathrm{~min}$ more in meal preparation and spend 9.5 min less in eating.

These results indicate that there appear to be significant relationships between SNAP receipt, benefit level and how households spend time in food-related activities, as well as between food insecurity and time spent in foodrelated activities. Predictably, these relationships differ depending on whether a household is headed by a single adult or a married couple.

\section{Discussion}

Our results indicate that time spent in household meal procurement is significantly related to household food security status and SNAP participation. However, the results differ between married and single households. 
In married households that participate in SNAP, less time is spent in meal preparation and shopping than in nonparticipating households. This may reflect Davis and You's finding that SNAP-participating households tend to be more time constrained than money constrained and, as a result, are unable to allocate sufficient time to meal production $^{(7)}$. In other words, although participating in SNAP assists households in surmounting financial barriers, the time barriers may remain.

Less time in meal preparation may also be a reflection of the work requirements associated with SNAP participation. In order to participate in SNAP, many states require households to either be employed part-time or participate in a work programme ${ }^{(16)}$. Consequently, these households have less time available to spend in meal preparation.

If married households that participate in SNAP spend less time in meal preparation than non-participating households, this may reveal an unforeseen challenge with SNAP. The TFP outlines low-cost diets for SNAP participants and determines the benefit level households receive. Food included in the TFP tends to be low-cost staples and ingredients that require cooking. In theory, if participant households follow this recipe guideline, these households can access sufficient food with their benefits. However, a number of studies have found that following the TFP requires a significant amount of time in meal preparation $^{(6)}$. Often households are unable to meet these time requirements. Davis and $\mathrm{You}^{(7)}$ found that a typical SNAP family following the TFP has a higher share of time cost of total production costs than the general population. Moreover, the TFP is more labour intensive than the actual amount of labour households allocate towards preparation. Davis and You argue that this can result in households purchasing more food away from home, which may, in turn, compromise the nutritional value of the meals households consume. In other words, the limited time households have to prepare food combined with the dietary guidelines of the TFP may be challenging for participating households.

In single households, SNAP participation and food insecurity are correlated with more time in meal preparation. Although this seems to contradict our assumption that single households are more time constrained, this may be reflective of single-parent households where household heads have no other adults with whom to share household responsibilities, including meal preparation.

On the other hand, a number of studies have found that time spent in food preparation can reduce the overall cost of production ${ }^{(1,17)}$. Single-headed households tend to have lower incomes than married households ${ }^{(18)}$. Although Davis and You found that households tend to spend more money on food than the TFP requires in order to make up for lack of time, it is possible that single households have budgets that do not allow them to replace food-related time with ingredients or other inputs. In fact, Hamermesh ${ }^{(19)}$ argues that it is difficult to substitute time for ingredients and other inputs and ingredients for time. Because of limited budgets, households may purchase cheap staples and whole foods and therefore devote more time to preparation than households with higher budgets.

While married and single households differ in how they allocate time to food preparation, both sets of households spend less time eating if the households are SNAP participants or food insecure. Returning to Davis and You's argument that the TFP requires excessive time for meal preparation, our results may indicate that households may consume more ready-to-eat meals acceptable by SNAP. A recent study found that households with income at 50 to $75 \%$ of the Federal Poverty Guideline spend a total of 48 cents/dollar of their food budget on foods away from home as well as frozen or prepared food ${ }^{(20)}$. These meals are often easier to eat 'on the run' thereby decreasing the amount of time spent eating.

Our paper has an empirical limitation. Because Coarsened Exact Matching does not remedy the selection problem inherent in SNAP and food insecurity studies, our results reflect correlations rather than an underlying causal relationship. Results indicate that food insecurity and SNAP participation are significant predictors of how households allocate their time, but they do not allow us to conclude that food insecurity or SNAP participation causes differential food-related time allocations. We performed a robustness check by running the identical regression models using a more comprehensive data set that includes the ATUS, FSS and the Current Population Survey's Annual Social and Economic Supplement, which contains information on SNAP participation and benefit level but no information on food insecurity. Our results for these regression models were similar to our original analysis and are available upon request.

Our results indicate a relationship between SNAP participation, food insecurity and time spent in food-related activities. Single households spend more time in food preparation and less time eating when participating in SNAP or when food insecure. Married households spend less time in meal preparation when participating in SNAP. Although a causal relationship was not established, the results highlight the relationship between food-related activities and programme participation and call for policies that address households that face time constraints as well as budget constraints.

\section{Practical and policy implications}

The results appear to indicate that federal nutrition programmes aimed at eliminating food insecurity should consider ways to support recipient meal procurement and preparation time constraints when creating and reforming nutrition education and policy. 
While SNAP nutritional educational programming is optional, these study results identify opportunities for consideration for food assistance programmes like SNAP to expand upon their educational programming to include time management skills, especially related to shopping, meal planning and meal preparation. However, education efforts alone will not likely have real meaningful impact. In order to further assist low-income households facing time constraints as well as financial barriers, federal food assistance programmes may have to creatively refashion assistance policy. For example, the Healthy, Hunger-Free Kids Act of 2010 (Public Law 111-296) calls for coordination across food assistance programmes. Recipient time could potentially be saved, for example, by establishing enrolment and recertification linkages across the National School Lunch Program, WIC (the Special Supplemental Nutrition Program for Women, Infants, and Children) and SNAP; and adopting 'area $v$. individual' eligibility. Implementation of large-scale policy efforts like these could result in instrumental support for time-strapped families and potentially cut administration costs. In the current economic downturn, it is important for policy makers and programme administrators to fully understand participant needs so they can better serve those experiencing food-related hardship. For SNAP, this is especially relevant given that each \$US 5 of federal SNAP benefits spent generates nearly twice that in local economic activity ${ }^{(21)}$; supporting the programme may be more palatable to decision makers during these difficult financial times.

\section{Acknowledgements}

Sources of funding: This research received no specific grant from any funding agency in public, commercial or not-for-profit sectors. Conflicts of interest: There are no conflicts of interest. Ethics: Ethical approval was not required for this study. Authors' contributions: T.K.M.B. conceptualized the idea, provided guidance on data, analysis and interpretation, and edited drafts. M.S.N. edited drafts and wrote the policy/implication section. C.T. contributed to the idea, harmonized the data, performed the data analysis and edited drafts.

\section{References}

1. Mancino L \& Newman C (2007) Who Has Time To Cook? How Family Resources Influence Food Preparation. Economic Research Report no. ERR-40. Washington, DC: Economic Research Service, USDA.

2. Stretcher V, McEvoy B, Becker M et al. (1986) The role of self-efficacy in achieving health behavior. Health Educ Behav 13, 73-92.

3. Luszczynska A, Tryburcy M \& Schwarzer R (2007) Improving fruit and vegetable consumption: a self-efficacy intervention compared with a combined self-efficacy and planning intervention. Health Educ Res 22, 630-638.

4. Anderson B, Winett RA \& Wojcik JR (2011) Social cognitive determinants of nutrition and physical activity among web-health users enrolling in an online intervention: the influence of social support, self-efficacy, outcome expectations, and self-regulation. J Med Internet Res 13, e28.

5. Seligman H, Davis TC, Schillinger D et al. (2010) Food insecurity is associated with hypoglycemia and poor diabetes self-management in a low income sample with diabetes. J Health Care Poor Underserved 21, 1227-1233.

6. Rose D (1999) Economic determinants and dietary consequences of food insecurity in the United States. J Nutr 129, 2S Suppl., 517S-520S.

7. Davis G \& You W (2010) The time cost of food at home: general and food stamp participant profiles. Appl Econ $\mathbf{4 2}$, $2537-2552$.

8. Hamermesh D (2008) Direct estimates of household production. Econ Lett $\mathbf{9 8}, 31-34$.

9. Abraham KG, Flood SM, Sobek M et al. (2011) American Time Use Survey Data Extract System: Version 2.4. College Park, MD and Minneapolis, MN: Maryland Population Research Center, University of Maryland and Minnesota Population Center, University of Minnesota.

10. US Census Bureau (2010) Current Population Survey Food Security Supplement Public Use File. Washington, DC: US Census Bureau.

11. Crossley T \& Lu Y (2004) Exploring the Returns to Scale in Food Preparation (Baking Penny Buns at Home). Hamilton, Ontario: McMaster University, Social and Economic Dimensions of an Aging Population Research Program.

12. Gundersen C \& Oliveira V (2001) The food stamp program and food insufficiency. Am J Agric Econ 84, $875-887$.

13. Nord M \& Golla AM (2009) Does SNAP Decrease Food Insecurity? Untangling the Self-Selection Effect. Economic Research Report no. ERR-85. Washington, DC: Economic Research Service, USDA.

14. Iacus SM, King G \& Porro G (2008) Matching for Causal Inference Without Balance Checking. Working Paper. Cambridge, MA: Harvard University.

15. Ho D, Imai K, King G et al. (2011) MatchIt: nonparametric preprocessing for parametric causal inference. J Stat Software 42, issue 8, 1-28; available at http://imai.princeton.edu/ research/files/matchit.pdf

16. US Department of Agriculture, Food and Nutrition Service (2012) Supplemental Nutrition Assistance Program: Employment Requirements. http://www.fns.usda.gov/ snap/applicant_recipients/employ_require.htm (accessed October 2012).

17. Rose D (2007) Food stamps, the Thrifty Food Plan and meal preparation: the importance of the time dimension for US Nutrition Policy. J Nutr Educ Behav 39, 226-232.

18. Sigle-Rushton W \& McClanahan S (2002) For richer or poorer: marriage as poverty alleviation in the United States. Population 57, 509-528.

19. Hamermesh DS (2007) Time to eat: household production under increasing income inequality. Am J Agric Econ 89, 852-863.

20. Frazao E, Andrews M, Smallwood D et al. (2007) Food Spending Patterns in Low Income Housebolds: Will Increasing Purchasing Power Result in Healthier Food Choices? Economic Information Bulletin no. EIB-29-4. Washington, DC: Economic Research Service, USDA.

21. Hanson K (2010) The Food Assistance National Input-Output Multiplier Model and Stimulus Effects of SNAP. Economic Research Report no. ERR-103. Washington, DC: Economic Research Service, USDA. 